\title{
Erratum
}

\section{Uniform Approximation by Meromorphic Functions Having Prescribed Poles}

\author{
Stephen Scheinberg
}

Math. Ann. 243, 83-93(1979)

I am indebted to Paul Gauthier for pointing out that the proof of Lemma 13 of the above-cited paper is incorrect as written. The following will correct the proof.

Suppose $X$ is a compact Riemann surface, $Y$ is an open subset of $X$ which we may think of as a possibly disconnected Riemann surface, and $Z$ is a relatively closed subset of $Y$. Denote by $\partial_{X}^{\infty} Z$ the compact set $\bar{Z}-Z=\bar{Z}-Y=\partial Z-Z$, where closures and boundaries are relative to $X$. Thus, the points of $\partial_{X}^{\infty} Z$ are at $\infty$ as far as the set $Z$ and the surface $Y$ are concerned. In the proof of Lemma 13 the symbol " $\partial$ " should be written in place of " $\partial$ " wherever the latter occurs. 